\title{
Article
}

\section{Observational signatures of outbursting protostars - II: Exploring a wide range of eruptive protostars}

MacFarlane, Benjamin, Stamatellos, Dimitris, Johnstone, Doug, Herczeg, Gregory, Baek, Giseon, Vivien Chen, Huei-Ru, Kang, SungJu and Lee, Jeong-Eun

Available at http://clok.uclan.ac.uk/28935/

MacFarlane, Benjamin, Stamatellos, Dimitris ORCID: 0000-0002-4502-8344, Johnstone, Doug, Herczeg, Gregory, Baek, Giseon, Vivien Chen, Huei-Ru, Kang, Sung-Ju and Lee, Jeong-Eun (2019) Observational signatures of outbursting protostars - II: Exploring a wide range of eruptive protostars. Monthly Notices of the Royal Astronomical Society, 487 (4). pp. 5106-5117. ISSN 0035-8711

It is advisable to refer to the publisher's version if you intend to cite from the work. http://dx.doi.org/10.1093/mnras/stz1570

For more information about UCLan's research in this area go to

http://www.uclan.ac.uk/researchgroups/ and search for < name of research Group>.

For information about Research generally at UCLan please go to http://www.uclan.ac.uk/research/

All outputs in CLoK are protected by Intellectual Property Rights law, including Copyright law. Copyright, IPR and Moral Rights for the works on this site are retained by the individual authors and/or other copyright owners. Terms and conditions for use of this material are defined in the policies page.

\section{CLoK}

Central Lancashire online Knowledge www.clok.uclan.ac.uk

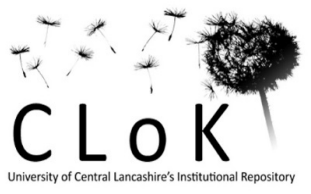




\title{
Observational signatures of outbursting protostars - II. Exploring a wide range of eruptive protostars
}

\author{
Benjamin MacFarlane, ${ }^{1}$ Dimitris Stamatellos ${ }^{\bullet},{ }^{1 \star}$ Doug Johnstone, ${ }^{2,3}$ \\ Gregory Herczeg, ${ }^{4}$ Giseon Baek, ${ }^{5}$ Huei-Ru Vivien Chen, ${ }^{6}$ Sung-Ju Kang ${ }^{7}$ and \\ Jeong-Eun Lee ${ }^{5}$ \\ ${ }^{1}$ Jeremiah Horrocks Institute for Mathematics, Physics and Astronomy, University of Central Lancashire, Preston PR1 2HE, UK \\ ${ }^{2}$ NRC Herzberg Astronomy and Astrophysics, 5071 West Saanich Rd, Victoria, BC V9E 2E7, Canada \\ ${ }^{3}$ Department of Physics and Astronomy, University of Victoria, Victoria, BC V8P 1A1, Canada \\ ${ }^{4}$ Kavli Institute for Astronomy and Astrophysics, Peking University, Yiheyuan 5, Haidian Qu, 100871 Beijing, China \\ ${ }^{5}$ School of Space Research and Institute of Natural Sciences, Kyung Hee University, 1732 Deogyeong-daero, Giheung-gu, Yongin-si, Gyeonggi-do 446-701, \\ Republic of Korea \\ ${ }^{6}$ Department of Physics and Institute of Astronomy, National Tsing Hua University, Kuang Fu Road, Hsinchu 30013, Taiwan \\ ${ }^{7}$ Korea Astronomy and Space Science Institute, 776 Daedeokdae-ro, Yuseong-gu, Daejeon 34055, Republic of Korea
}

Accepted 2019 June 2. Received 2019 May 29; in original form 2019 April 27

\begin{abstract}
Young stars exhibit variability due to changes in the gas accretion rate onto them, an effect that should be quite significant in the early stages of their formation. As protostars are embedded within their natal cloud, this variability may only be inferred through long wavelength observations. We perform radiative transfer simulations of young stellar objects (YSOs) formed in hydrodynamical simulations, varying the structure and luminosity properties in order to estimate the long-wavelength, sub-mm and $\mathrm{mm}$, variations of their flux. We find that the flux increase due to an outburst event depends on the protostellar structure and is more prominent at sub-mm wavelengths than at $\mathrm{mm}$ wavelengths; e.g. a factor of 40 increase in the luminosity of the young protostar leads to a flux increase of a factor of 10 at $250 \mu \mathrm{m}$ but only a factor of 2.5 at $1.3 \mathrm{~mm}$. We find that the interstellar radiation field dilutes the flux increase but that this effect may be avoided if resolution permits the monitoring of the inner regions of a YSO, where the heating is primarily due to protostellar radiation. We also confirm that the bolometric temperature and luminosity of outbursting protostars may result in an incorrect classification of their evolutionary stage.
\end{abstract}

Key words: accretion, accretion discs - radiative transfer-stars: protostars - stars: variables: general.

\section{INTRODUCTION}

Young protostars are believed to grow in mass through episodes of intense gas accretion that result in luminosity outbursts, as seen e.g. in FU Ori-type (Herbig 1966, 1977) and EXOr-type objects (Herbig 1989, 2008). In the last decade there has been an increased interest in the variability of eruptive young stars and its role in star formation (see Audard et al. 2014, for a review). Such strong variability (of a few magnitudes) at the earliest phases of star formation could explain the luminosity problem (Kenyon et al. 1990), i.e. the fact that the observed luminosity of young stars is too low compared to what is expected from the gas accretion rate that is needed to accumulate their mass within the required timeframe (Enoch et al. 2009; Evans et al. 2009; Dunham et al. 2015)

It is expected that luminosity outbursts are more prominent during the initial phases of star formation Class 0, I objects) but only a few observations of varying YSOs have been reported (Caratti o Garatti et al. 2011; Kóspál et al. 2011; Safron et al. 2015). This may be due to (i) the short duration of the these early phases of star formation, or (ii) the short duration of an outburst episode (a few years to a few centuries). Furthermore, as young protostars are deeply embedded in their parent envelopes the effect of an outburst may be difficult to observe, at least at optical wavelengths. Therefore, at longer wavelength, observations may be more appropriate (e.g. Kóspál et al. 2007; Safron et al. 2015; Hunter et al. 2017; Yoo et al. 2017)

Recognizing the importance of longer wavelength observations, the James Clerk Maxwell Telescope (JCMT) TRANSIENT Survey 
is monitoring eight star forming regions within $500 \mathrm{pc}$ (Herczeg et al. 2017), with the goal to observe variability at sub-mm wavelengths related to episodic accretion events. Johnstone et al. (2018) summarize the results of the survey, which has observed significant variability in EC53 (Yoo et al. 2017) and small changes on other objects (Mairs et al. 2018).

MacFarlane et al. (in preparation) (hereafter Paper I), investigate the effect of episodic outbursts on the spectral energy distribution (SED) of a YSO self-consistently formed within a hydrodynamic simulation of a collapsing cloud, including episodic accretion leading to episodic luminosity outbursts. They performed polychromatic radiative transfer calculations using the properties (luminosity, density structure) of the young protostar as provided by the simulation. Here, in order to compare against a broader variety of outbursting young stars (Audard et al. 2014), we perform an additional set of 25 radiative transfer (RT) simulations, exploring a wider parameter space of outburst luminosities and YSO properties (disc mass, envelope mass, and outflow cavity).

This paper is structured as follows. In Section 2 we briefly describe the hydrodynamic simulation and the radiative transfer method used. In Section 3 we discuss the effect of outbursts on the SED for different outburst luminosities and YSO structures. Finally, in Section 4 we summarize our results.

\section{COMPUTATIONAL METHOD}

We briefly describe the hydrodynamic simulation and the radiative transfer method. For a more detailed discussion, please see Paper I. We then discuss how we modify the properties of the YSO to cover a more extended parameter space.

\subsection{Hydrodynamic simulation of YSO formation}

We follow the evolution of a collapsing $5.4 \mathrm{M}_{\odot}$ cloud that forms an embedded protostar (Stamatellos, Whitworth \& Hubber 2012), using the Smoothed Particle Hydrodynamics (SPH) code SEREN (Hubber et al. 2011a,b) with the radiative transfer method of Stamatellos et al. (2007). If we assume a typical star formation efficiency of $\sim 20$ per cent (e.g. André et al. 2014) then this $5.4 \mathrm{M}_{\odot}$ cloud will end up forming roughly a solar-mass star. The simulation employs a model to capture the episodic accretion of material onto the young protostar that results in episodic outbursts of luminosity (for details see Paper I Stamatellos, Whitworth \& Hubber 2011; Stamatellos et al. 2012), and in turn affecting the structure of the YSO (MacFarlane \& Stamatellos 2017). The episodic behaviour is due to the effect of gravitational instability (GI) operating in the outer disc, combined with the magnetorotational instability (MRI) operating in the inner disc when the temperature is high enough to provide an appropriate level of ionization (Armitage, Livio \& Pringle 2001; Zhu et al. 2010a; Zhu, Hartmann \& Gammie 2010b; Stamatellos et al. 2011; Mercer \& Stamatellos 2017).

\subsection{Radiative transfer modelling}

We perform detailed polychromatic radiative transfer simulations using the $3 \mathrm{D}$ radiative transfer Monte Carlo code RADMC-3D 1 (Dullemond et al. 2012). This code utilizes a Ray-tracing Radiative Transfer (RRT), to produce synthetic observations (SEDs and

${ }^{1}$ http://www.ita.uni-heidelberg.de/ dullemond/software/radmc-3d/ (Last accessed: 07/07/2018)
Table 1. Parameters for the $25 \mathrm{RT}$ models. The outflow cavity opening angle at $r_{x y}=10000 \mathrm{AU}$ is set to $\theta_{\mathrm{c}}=10^{\circ}$ for all models. Disc $\left(M_{\mathrm{d}}\right)$ and envelope $\left(M_{\mathrm{e}}\right)$ masses are given in solar masses and in units of the corresponding masses from the simulation $\left(M_{\mathrm{d}}^{\mathrm{s}} \simeq 0.3 \mathrm{M}_{\odot} ; M_{\mathrm{e}}^{\mathrm{s}} \simeq 4 \mathrm{M}_{\odot}\right)$. The quiescent phase snapshot of event E2 is used (where $L_{*}=2 \mathrm{~L}_{\odot}$; see Paper I).

\begin{tabular}{lcccc}
\hline$M_{\mathrm{d}} / M_{\odot}$ & $M_{\mathrm{d}} / M_{\mathrm{d}}^{\mathrm{s}}$ & $M_{\mathrm{e}} / M_{\odot}$ & $M_{\mathrm{e}} / M_{\mathrm{e}}^{\mathrm{s}}$ & $L_{*}$ \\
\hline 0.3 & 1 & 4 & 1 & $2,10,20,50,80$ \\
0.03 & 0.1 & 4 & 1 & $2,10,20,50,80$ \\
0.6 & 2 & 4 & 1 & $2,10,20,50,80$ \\
0.3 & 1 & 0.4 & 0.1 & $2,10,20,50,80$ \\
0.3 & 1 & 8 & 2 & $2,10,20,50,80$ \\
\hline
\end{tabular}

images) that can then be compared with observations. To translate the SPH density profile to a grid (Stamatellos \& Whitworth 2005) to be used by RADMC-3D an adaptive-mesh refinement method is used (Robitaille 2011; see Paper I). Our models take into account radiation from the young protostar (both intrinsic and due to accretion) and heating from the ambient interstellar radiation field (ISRF) (André et al. 2004). We assume a gas-to-dust ratio of 100, and we use the Ossenkopf \& Henning (1994) (OH5) opacities that refer to a standard MRN mixture (Mathis, Rumpl \& Nordsieck 1977; 53 per cent silicate, 47 per cent graphite) with thin ice mantles at a density of $10^{6} \mathrm{~cm}^{-3}$.

\subsection{YSO model setup}

The parameters of the RT models performed are presented in Table 1. We chose an accretion event from the hydrodynamic simulation (event E2; see Paper I) and we perform RT simulations on two snapshots: just before and near the end of the outburst event. The mass of the protostar at the two chosen snapshots are 0.26 and $0.42 \mathrm{M}_{\odot}$, respectively, and the corresponding luminosities are $2.0 \mathrm{~L}_{\odot}$ and $1110 \mathrm{M}_{\odot}$. The high luminosity provided by the hydrodynamic simulation is due to an intense accretion event. Such events are thought to be rather common at the early phases of the formation of the protostar, and indeed high outburst luminosities have been observed e.g. Z CMa (400-600 L $\odot$; Hartmann \& Kenyon 1996) and V1057 Cyg (250-800 L $\odot$; Hartmann \& Kenyon 1996). We artificially set the outburst luminosities to $10,20,50$, and $80 \mathrm{~L}_{\odot}$, rather than $1110 \mathrm{~L}_{\odot}$ as directly provided by the hydrodynamic simulation (see Paper I). These luminosities have been chosen so as to correspond to the wide range of the luminosities of FUOri type outbursts observed (Audard et al. 2014), which cannot be captured by a single hydrodynamic simulation. The quiescent phase protostellar luminosity is that of the simulation output, i.e. $L_{*, q}=2 \mathrm{~L}_{\odot}$. The ratios of protostellar luminosity between the outbursting and the quiescent phase are therefore 5,10, 25, and 40 , respectively. We therefore make the inherent assumption that the density profile of the YSO does not depend on the luminosity of the embedded protostar, which is not true (MacFarlane \& Stamatellos 2017). However, our aim here is to investigate how radiation propagates (and eventually reaches the observer) through an asymmetric YSO, using a given density structure (provided by the hydrodynamic simulation). Moreover, the luminosity of the protostar affects mostly the density structure near it whereas the large-scale structure remains mostly unaffected (see MacFarlane \& Stamatellos 2017, for details).

In addition to varying the outburst luminosity, we also vary the mass of the different components of the YSO, to explore their impact on the SED during an episodic accretion event. A schematic 


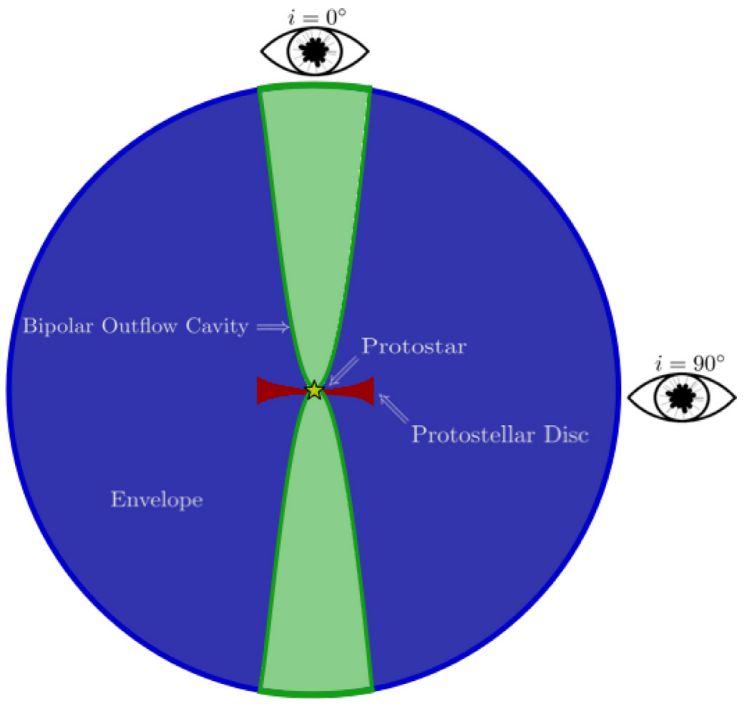

Figure 1. Schematic representation of the different components of the modelled YSO: disc, envelope, and outflow cavity. The inclinations $i=$ $0^{\circ}$ and $90^{\circ}$ are also indicated.

representation of the different components (disc, envelope, and outflow cavity) of the YSO considered is presented in Fig. 1. We perform RT simulations of a YSO for which the disc and envelope densities are each rescaled to $\times 0.1$ and $\times 2$ times, respectively, those of the simulation output. The disc-envelope radial boundary is set by the location at which the surface density falls below a value of $20 \mathrm{gcm}^{-2}$. The vertical boundary of the disc is set to three times the disc scale height $h_{\mathrm{d}}\left(r_{x y}\right)$, where

$h_{\mathrm{d}}\left(r_{x y}\right)=\frac{c_{s}\left(r_{x y}\right)}{\Omega_{K}\left(r_{x y}\right)}$.

In the above formalism, $r_{x y}$ is the radius on the disc mid-plane, $c_{s}\left(r_{x y}\right)$ is the sound speed, and $\Omega_{K}\left(r_{x y}\right)$ is the Keplerian angular velocity.

Additionally, the model accounts for bipolar outflows in the direction perpendicular to the disc mid-plane. We define the surface of the outflow cavity using

$\left|z\left(r_{x y}\right)\right|=\alpha r_{x y}^{\beta}$,

where $\alpha$ and $\beta$ are free parameters that determine the cavity opening angle $\theta_{\mathrm{c}}$ and the cavity flaring $\left(\tan \theta_{\mathrm{c}}=\alpha\left(r_{x y}^{\mathrm{c}}\right)^{\beta-1}\right)$, and $r_{x y}^{\mathrm{c}}$ is the radius on the disc mid-plane at which $\theta_{\mathrm{c}}$ is calculated. The density and geometry (i.e. the values of $\alpha, \beta$ above) of the outflow cavity depend on the evolutionary stage of the YSO. As we focus on Class 0 YSOs, motivated by the works of Whitney et al. (2003a,b), we set $\beta=3$, and the opening angle of the cavity to $\theta_{\mathrm{c}}=10^{\circ}$ at $r_{x y}^{\mathrm{c}}=10000 \mathrm{AU}$. The cavity density is set to $n_{\mathrm{H}_{2}}=10^{5} \mathrm{~g} \mathrm{~cm}^{-3}$. At large distances from the star, we ensure that the density of the cavity is not higher than the density of the adjacent envelope by tapering this value.

\section{RESULTS}

\subsection{Spectral Energy Distributions}

For each model, we compute SEDs for inclinations of $0^{\circ}, 30^{\circ}$, $60^{\circ}$, and $90^{\circ}$, for radiation originating from $R \leq 10000 \mathrm{AU}$. This is the typical size of a Class 0 object as mapped in the sub-mm (e.g. at $850 \mu \mathrm{m}$ by JCMT). In Section 3.4 we also also present

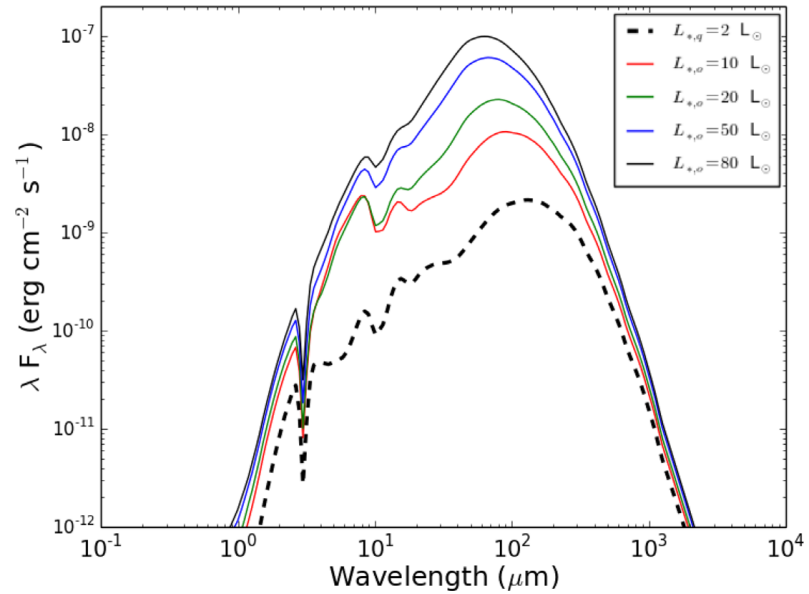

Figure 2. SEDs for models with different protostellar luminosities, for unscaled YSOs at an inclination of $i=30^{\circ}$. The thick black dashed line corresponds to the quiescent phase $\left(L_{*, q}=2 \mathrm{~L}_{\odot}\right)$. The solid red, green, blue, and black lines represent outburst phases of the YSO, with protostellar luminosities of $L_{*, o}=10,20,50$, and $80 \mathrm{~L}_{\odot}$, respectively.

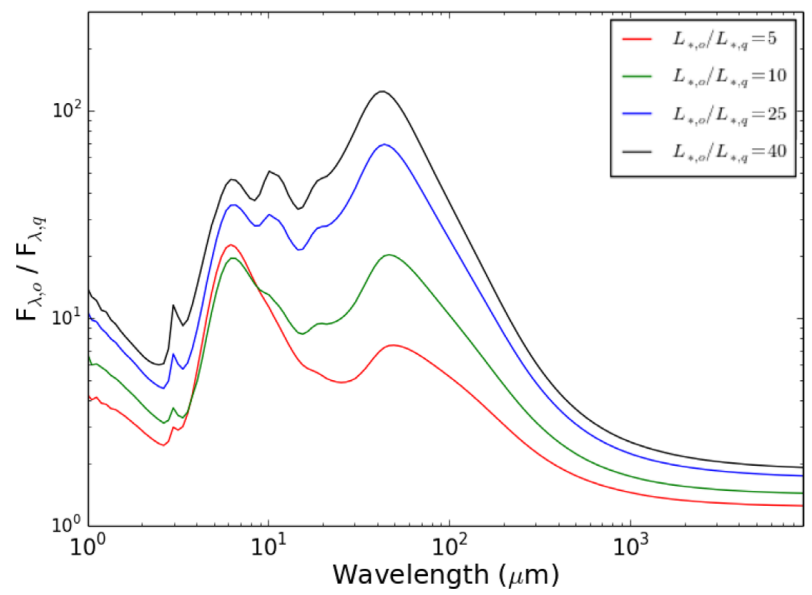

Figure 3. The ratio of outburst to quiescent flux for the RT models presented in Fig. 2. The flux increase is more prominent in the NIR but still present at the FIR and submm/mm region.

RT models with integration areas $R \leq 1000 \mathrm{AU}$, corresponding to the central regions of YSOs. SEDs are computed assuming a distance of 140 pc. In Fig. 2 we present SEDs for RT models with unscaled disc and envelope components. SEDs are computed at an inclination of $i=30^{\circ}$, and in Fig. 3 we present the ratio between the outburst and the quiescent flux. We see that the maximum increase of flux occurs around $5-80 \mu \mathrm{m}$ (a factor from $\sim 10$ to 100 for a luminosity increase of a factor 5-40). However, there is also a detectable increase at longer wavelengths (e.g. $\sim 1.5-3$ at $850 \mu \mathrm{m})$.

In Fig. 4, we present the SEDs for a representative model with an outbursting phase luminosity of $L_{*, o}=10 \mathrm{~L}_{\odot}$, at an inclination of $i=30^{\circ}$. Models with rescaled envelope mass $(\times 0.1, \times 1$ unscaled, and $\times 2$ the original value) are plotted. As expected, with decreased envelope mass the flux increases at short wavelengths (below around 100-200 $\mu \mathrm{m}$ ) and decreases at longer wavelengths. Likewise, increasing the envelope mass results in the reduction of short-wavelength flux and increase of flux at longer wavelengths. 


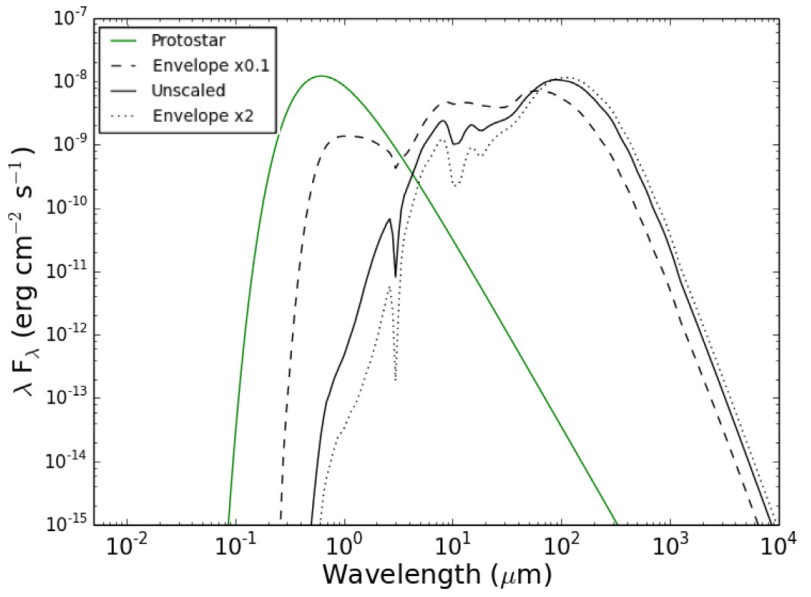

Figure 4. SEDs of YSOs with varying envelope mass for protostellar luminosity $L_{*, o}=10 \mathrm{~L} \odot_{\odot}$. The black dashed, solid, and dotted lines represent models where the envelope mass is scaled by $\times 0.1, \times 1$, and $\times 2$, respectively. SEDs are computed at an inclination of $30^{\circ}$. The green solid line corresponds to the protostellar emission.

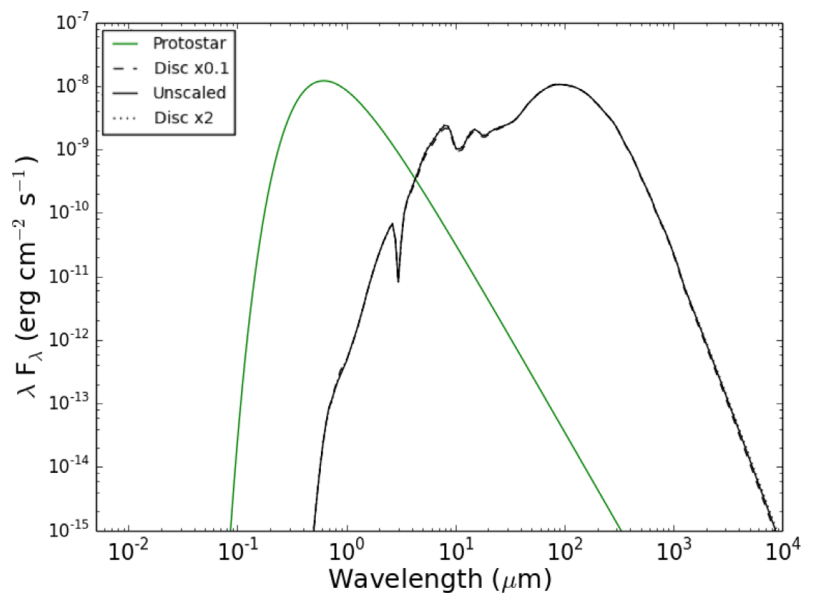

Figure 5. As per Fig. 4, but for varying disc mass. The black dashed, solid, and dotted lines correspond to models with disc masses scaled to $\times 0.1, \times 1$, and $\times 2$ the hydrodynamic simulation value, respectively.

We also present SEDs comparing models with rescaled disc mass in Fig. 5. The impact of disc mass rescaling is minimal when compared to the envelope-rescaled models. This is because the rescaled disc mass is of the order of $0.3 \mathrm{M}_{\odot}$, whereas the envelope mass is of the order of $4 \mathrm{M}_{\odot}$. The change in the total mass is therefore much larger when the mass of the envelope is scaled. It is expected that the effect of the disc will be more prominent when viewing the disc edge-on.

\subsection{Flux increases during outbursts}

For each outburst phase protostellar luminosity, we compute the outbursting-to-quiescent flux ratios at 70, 250, 850, and $1300 \mu \mathrm{m}$, i.e. wavelengths corresponding to continuum bands of the Herschel Space Observatory, JCMT, and Atacama Large (Sub-)Millimetre Array (ALMA). By computing the flux response due to a known increase in protostellar luminosity, we provide a diagnostic tool for future observations to determine the protostellar luminosity increases responsible for increases to long wavelength emission.
Flux ratios are plotted for all models in Fig. 6, for $70 \mu \mathrm{m}$ (top left panel), $250 \mu \mathrm{m}$ (top right panel), $850 \mu \mathrm{m}$ (bottom left panel), and $1300 \mu \mathrm{m}$ (bottom right panel). We find that the flux ratio increases at all wavelengths with increasing ratio of outburstingto-quiescent protostellar luminosity, but that the flux increase is smaller at longer wavelengths. Illustrating this further, we refer to Figs 2-3, where we see that between the quiescent phase (dashed line) and outburst phase (solid lines) SEDs, the greatest increase in flux occurs around $5-80 \mu \mathrm{m}$. For the flux ratios at 70 and $250 \mu \mathrm{m}$, the response to the protostellar luminosity increase is not significantly different for models with different disc masses. On the other hand, higher envelope masses result in higher flux ratios. The contribution from the disc is rather small, considering that it has much smaller mass than the envelope itself. For the flux ratios at 850 and $1300 \mu \mathrm{m}$, there are only small differences between different models.

\subsection{YSO classification}

We compute bolometric values for the different YSOs and in Fig. 7 we plot the bolometric luminosity $L_{\mathrm{BOL}}$ against the bolometric temperature $T_{\mathrm{BOL}}$ (Myers \& Ladd 1993). Our aim is to consider the impact of the varying protostellar luminosities and different masses of YSO components on the calculated values (and the subsequent classification of the YSO). We also plot the values derived from the YSO in the hydrodynamic simulation (see Paper I for description of the two events E1 and E2) (thin diamonds: E1; thick diamonds: E2). The vertical dashed line in Fig. 7 indicates the $T_{\mathrm{BOL}}$ boundary of $70 \mathrm{~K}$ between YSO Class 0 and I (Chen et al. 1995). The bolometric temperature generally increases with decreasing inclination, i.e. when looking through the outflow cavity, as the hotter regions close to the protostar are probed. The only exception is when the mass of the envelope is scaled down by a factor of 10; this is due to the envelope asymmetric structure which is more pronounced relative to the outflow cavity (in this model the density of the cavity is not much different from the density of envelope itself; also see the discussion on envelope asymmetries in Paper I).

All the different YSOs that we model here (Fig. 7) are by definition Class 0 objects as there is more mass in the envelope than in the protostar itself. However, we note that the models in which we decrease the envelope mass by a factor of 10 are on the boundary between Class 0 and Class I phase $\left(M_{*} \approx M_{\mathrm{e}}\right)$. The observed bolometric temperature is found to be sensitive to the YSO inclination, and it may change the classification from Class 0 to Class I if a strict value of $70 \mathrm{~K}$ is used. The influence of geometry on bolometric properties folding into YSO classification has been previously noted (e.g. Dunham \& Vorobyov 2012; Dunham et al. 2013, and Paper I). If we use a boundary value of $100 \mathrm{~K}$ instead of $70 \mathrm{~K}$ then most of the YSOs would be appropriately classified as Class 0 objects, with the exception of the low envelope-mass models that exhibit high bolometric temperatures and would be still classified as Class I objects (but nevertheless they are in the boundary between the two phases). However, the definition of a strict boundary is not possible without exploring a wider parameter space as there is a dependence on the YSO luminosity and orientation.

\subsection{Impact of the integration area}

High resolution observations (e.g. by ALMA) can probe the very central regions of YSOs, providing better constraints on the 

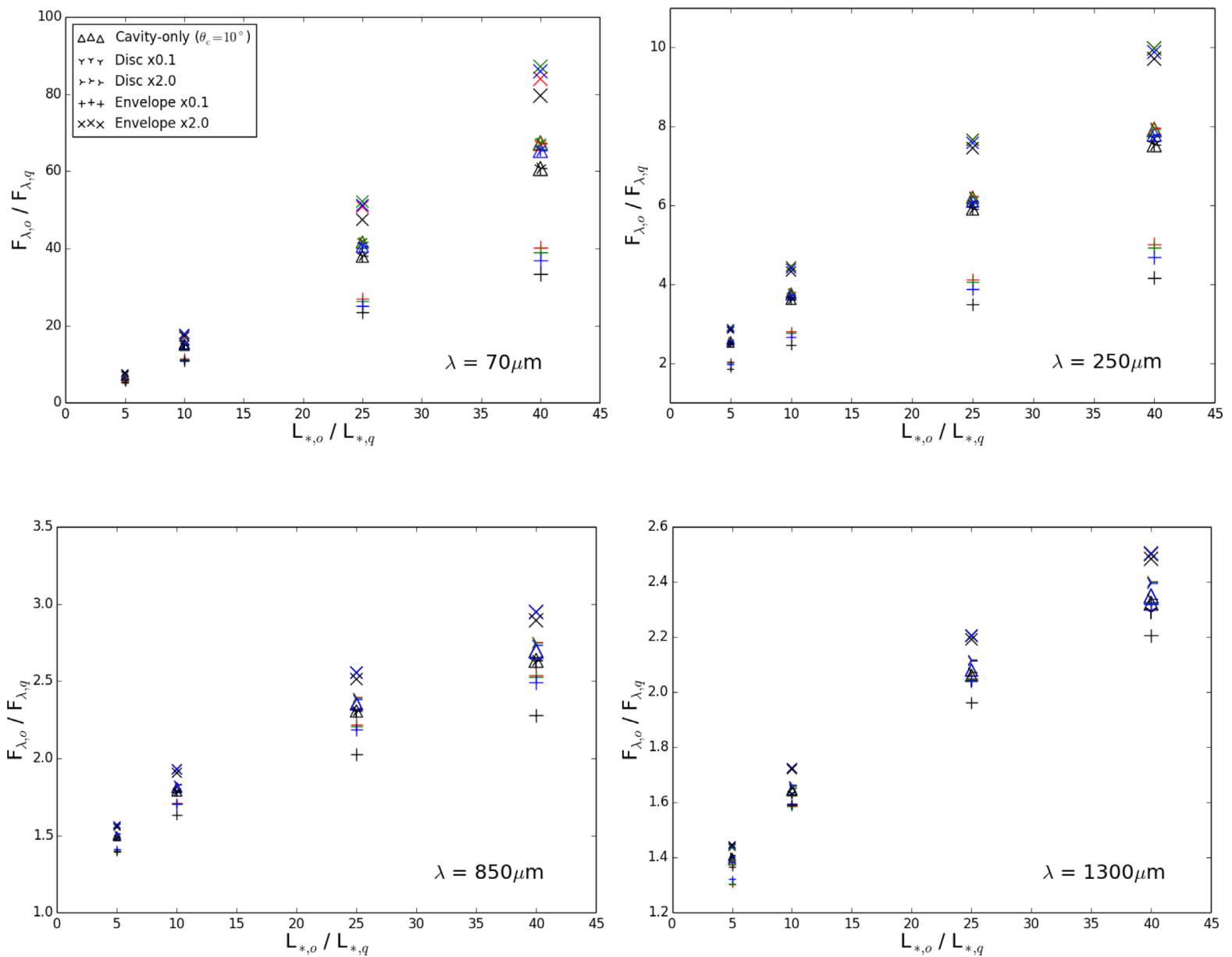

Figure 6. Outburst-to-quiescent flux ratios, $F_{\lambda, o} / F_{\lambda, q}$, at 70, 250, 850, and $1300 \mu \mathrm{m}$, as a function of the protostellar luminosity $L_{*, o}$, computed integrating over the inner $R \leq 10000 \mathrm{AU}$. The red, green, blue, and black markers correspond to SEDs at $0^{\circ}, 30^{\circ}, 60^{\circ}$, and $90^{\circ}$, respectively. The triangles represent the default model (no rescaling), upward/downward tri-spoke markers are models with disc masses rescaled to 0.1/2 times the simulation values, and plus/cross markers are models with envelope masses scaled 0.1/2 times.

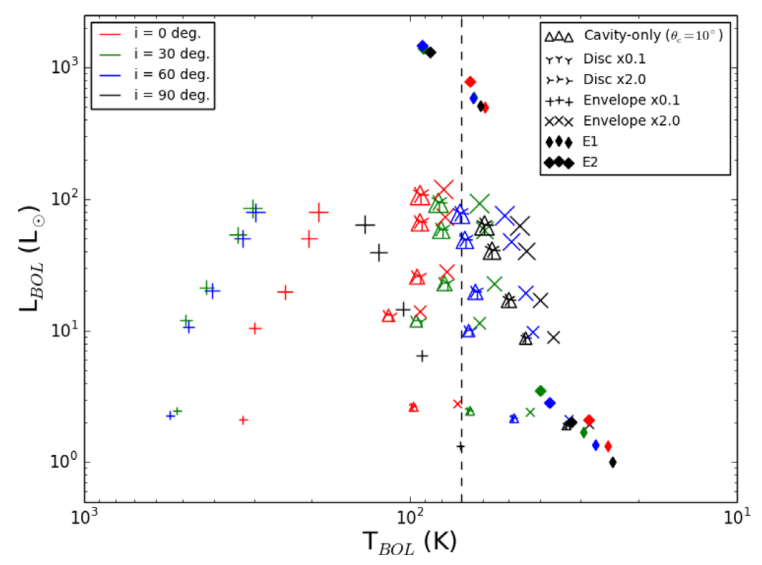

Figure 7. $L_{\mathrm{BOL}}$ as a function of $T_{\mathrm{BOL}}$. The markers and colours as per Fig. 6. The diamond markers denote data from models adopting quiescent/outbursting protostellar luminosities from the hydrodynamic simulation, where thin/thick markers are for E1/E2 events. The symbol sizes are proportional to the luminosity of the protostar. The dashed vertical line represents the Class 0/I YSO boundary. properties of embedded young protostars. We perform additional RT simulations, computing SEDs integrating over the inner $R \leq$ $1000 \mathrm{AU}$ region of the YSO, which corresponds to an angular size of 2-7 arcsec at typical distances of nearby star forming regions (500 to $140 \mathrm{pc}$ ). We then compute the flux ratios and discuss the corresponding $L_{\mathrm{BOL}}-T_{\mathrm{BOL}}$ diagnostics, to understand the impact of the integration area on the flux changes during outbursts, and on the YSO classification.

\subsubsection{Flux ratio changes}

We present the flux ratios between the quiescent and outbursting phases, at wavelengths of 70, 250, 850, and $1300 \mu \mathrm{m}$ in Fig. 8. We find a similar behaviour to the previous analysis (i.e. integrating over the inner $R \leq 10000 \mathrm{AU}$ region), i.e. all flux ratios increase with increasing outburst protostellar luminosity.

At 70 and $250 \mu \mathrm{m}$, we find that the flux ratios are smaller in comparison with those computed over a more extended region $(R \leq 10000 \mathrm{AU})$. They also exhibit smaller differences between models. With decreasing integration area over which the flux ratios is computed, the effect of varying the envelope mass becomes unimportant, as for the smaller region considered, the YSO mass is dominated by the disc mass. As the changes in the disc mass are relatively small between different models. 

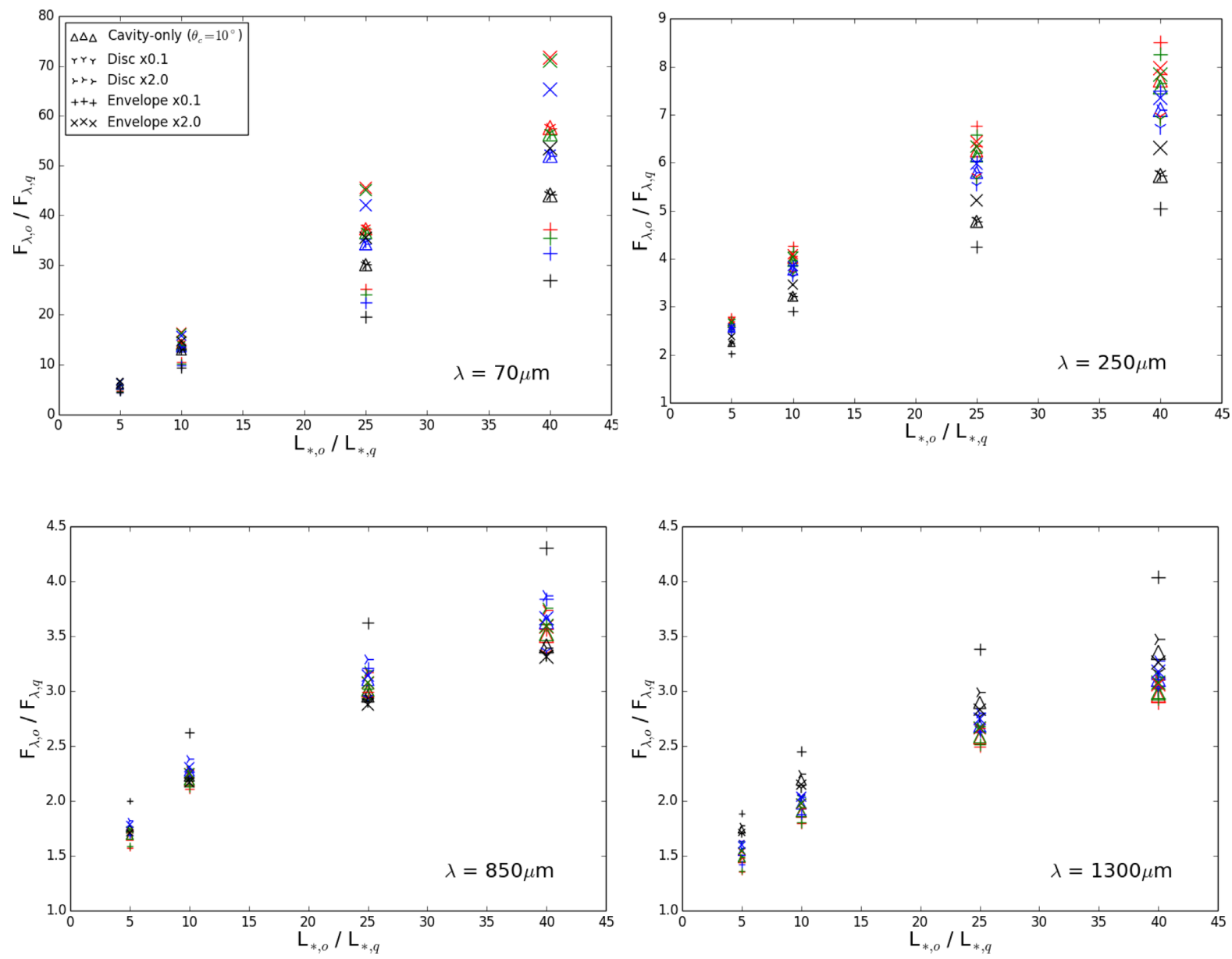

Figure 8. Outburst-to-quiescent flux ratios, $F_{\lambda, o} / F_{\lambda, q}$, at 70, 250, 850, and $1300 \mu \mathrm{m}$, as a function of the outburst protostellar luminosity $L_{*, o}$, computed integrating over the inner $R \leq 1000 \mathrm{AU}$ (symbols are the same as in Fig. 6).

It follows that for all models, more centrally located regions have increasingly similar masses, and therefore similar continuum fluxes.

Both the 850 and $1300 \mu \mathrm{m}$ continuum wavelengths exhibit higher flux ratio increases for SEDs computed over smaller spatial scales with the YSO (compare Fig. 6 with Fig. 8). This is a result of not including the outer regions of the envelope, which are primarily heated by the ISRF. The inclusion of the outer YSO regions attenuates the effect of the protostellar luminosity on the SEDs. The result shown here serves as a clear motivation to pursue high-resolution monitoring of episodically accreting protostars, particularly at (sub-) $\mathrm{mm}$ wavelengths, to ensure sufficient contrast in flux between quiescent and outbursting phases, whilst at the same time minimizing the effect of the ISRF.

\subsubsection{YSO classification for a smaller integration area}

We present $L_{\mathrm{BOL}}$ and $T_{\mathrm{BOL}}$ values for the SED integrated over the $R \leq 1000 \mathrm{AU}$ region in Fig. 9. Two clear trends are found when comparing with the results computed considering a larger integration area $\left(R \leq 10000 \mathrm{AU}\right.$; see Fig. 7): $T_{\mathrm{BOL}}$ increases, as we consider only the hotter inner region, while $L_{\mathrm{BOL}}$ decreases, as we do not take into account a significant portion of the radiation from the extended envelope. We find that by proxy of the $T_{\mathrm{BOL}}=$

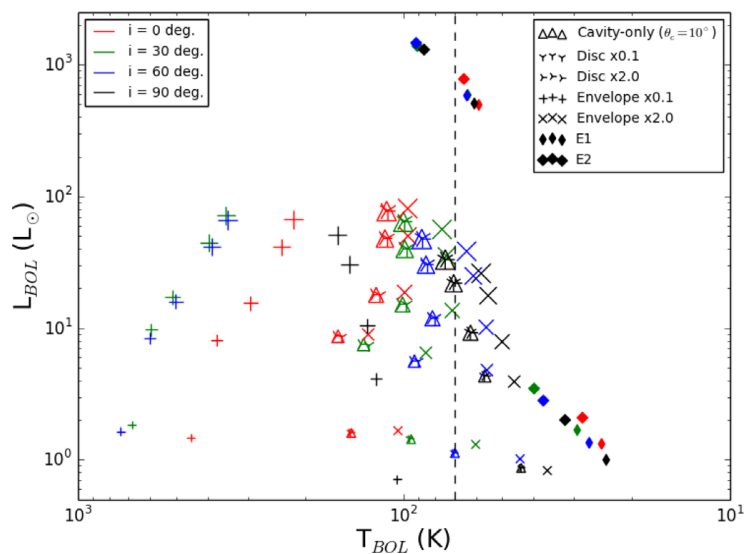

Figure 9. $L_{\mathrm{BOL}}$ as a function of $T_{\mathrm{BOL}}$ for the RT models in Table 1. Markers and colours as per Fig. 7. Figure as per Fig. 7, but the bolometric properties are computed including flux only from the inner $R \leq 1000 \mathrm{AU}$ region of the YSO.

$70 \mathrm{~K}$ boundary, all YSOs models in which the envelope mass is reduced are classified as Class I objects. Unscaled and discmass-scaled models at inclinations lower than $90^{\circ}$ also correspond to Class I YSOs. In contrast, the most heavily embedded (with twice as much mass in the envelope), and lowest luminosity, 
edge-on models with no scaling, or decreased/increased disc mass, are classified as Class 0. This result confirms that the SED integration area also affects the bolometric properties of YSOs.

\section{CONCLUSIONS}

We have explored a range of radiative transfer models of episodically outbursting YSOs with a variety of structure and luminosities, with the aim to provide a diagnostic study to estimate the change in luminosity of episodically outbursting embedded protostars using long wavelength observations.

We based our YSO models on the results of hydrodynamic simulations that include episodic feedback (see Paper I) but we extended the YSO parameter space by varying the protostellar luminosity and the masses of the different components of the YSO (disc, envelope, and outflow cavity) to compute SEDs over the central $R \leq 10000$ AU of the YSO at different inclinations. An additional set of SEDs were computed, integrating flux over the central $R \leq 1000$ AU region of the YSO.

We calculated the flux increase at different wavelengths during episodic accretion events, and we found a reduction in outburst-toquiescent flux ratio with increasing wavelength. Long wavelength emission shows only a small flux ratio increase during an outburst (an increase by a factor of 1.3 to 2.6 at $1.3 \mathrm{~mm}$ ). In contrast, there are more significant differences in the flux ratio at shorter (far-IR) wavelengths (an increase by a factor of 10 to 90 at $70 \mu \mathrm{m}$ ). This is similar to what is seen for EC53 (Yoo et al. 2017), and also for the outbursting protostar 2MASS $22352345+7517076$ that has been observed at many different wavelengths by various telescopes (Kun et al. 2019).

When the flux is computed over the $R \leq 1000$ AU region, it was found that there is only a moderate increase of the flux ratio at far-IR wavelengths (an increase by a factor of 5 to 70 at $70 \mu \mathrm{m}$ ). At (sub)mm wavelengths when integrating over a smaller region around the YSO, the flux ratio increase is larger than when integrating over an area of $10000 \mathrm{AU}$ (an increase by a factor of 1.5 to 4 at $1.3 \mathrm{~mm}$ ). This provides evidence that high-resolution observations (e.g. with ALMA; see Francis et al. 2019) can be used to provide more reliable estimates of the change of the intrinsic luminosity of protostars undergoing episodic accretion.

We calculated the bolometric luminosity and temperature of the YSOs of different models and found that the bolometric temperature is sensitive to the YSO inclination, and it may affect the classification of a YSO if a strict value of $70 \mathrm{~K}$ is used to distinguish between Class 0 and Class I objects. If we use a boundary value of $100 \mathrm{~K}$ instead of $70 \mathrm{~K}$ we obtain a more accurate classification.

The work presented in this paper describes the observational characteristics of young Class 0/I protostars undergoing episodic phases of mass accretion and therefore luminosity outbursts, akin to FU Ori-type objects. Our results provide diagnostics to infer the luminosity of episodically outbursting embedded protostars using observations at FIR and mm wavelengths.

\section{ACKNOWLEDGEMENTS}

BM is supported by STFC grant ST/N504014/1. DS is partly supported by STFC grant ST/M000877/1. DJ is supported by the National Research Council Canada and by an NSERC Discovery Grant. GH is supported by general grant 11773002 awarded by the National Science Foundation of China. Simulations were performed using the UCLAN HPC facility and the COSMOS Shared Memory system at DAMTP, University of Cambridge operated on behalf of the STFC DiRAC HPC Facility. This equipment is funded by BIS National E-infrastructure capital grant ST/J005673/1 and STFC grants ST/H008586/1, ST/K00333X/1. SEREN has been developed and maintained by David Hubber, who we thank for his help. Column density maps were generated using the visualization software SPLASH (Price 2007). This work is supported by the JCMT-Transient Team.

\section{REFERENCES}

André P., Bouwman J., Belloche A., Hennebelle P., 2004, Ap\&SS, 22, 325

André P., Di Francesco J., Ward-Thompson D., Inutsuka S.-I., Pudritz R. E., Pineda J. E., 2014, in Beuther H., Klessen R. S., Dullemond C. P., Henning T., eds, Protostars and Planets VI. University of Arizona Press, Tucson, p. 27

Armitage P. J., Livio M., Pringle J. E., 2001, MNRAS, 324, 705

Audard M. et al., 2014, in Beuther H., Klessen R. S., Dullemond C. P., Henning T., eds, Protostars and Planets VI. University of Arizona Press, Tucson, p. 387

Caratti o Garatti A. et al., 2011, A\&A, 526, L1

Chen H., Myers P. C., Ladd E. F., Wood D. O. S., 1995, ApJ, 445, 377

Dullemond C. P., Juhasz A., Pohl A., Sereshti F., Shetty R., Peters T., Commercon B., Flock M., 2012, RADMC-3D: A multi-purpose radiative transfer tool, Astrophysics Source Code Library, record ascl:1202.015

Dunham M. M., Vorobyov E. I., 2012, ApJ, 747, 52

Dunham M. M. et al., 2013, AJ, 145, 94

Dunham M. M. et al., 2015, ApJS, 220, 11

Enoch M. L., Evans N. J., II, Sargent A. I., Glenn J., 2009, ApJ, 692, 973

Evans N. J., II et al., 2009, ApJS, 181, 321

Francis L., Johnstone D., Dunham M. M., Hunter T. R., Mairs S., 2019, ApJ, 871,149

Hartmann L., Kenyon S. J., 1996, ARA\&A, 34, 207

Herbig G. H., 1966, Vistas Astron., 8, 109

Herbig G. H., 1977, ApJ, 217, 693

Herbig G. H., 1989, in Reipurth B., ed., European Southern Observatory Conference and Workshop Proceedings Vol. 33, European Southern Observatory Conference and Workshop Proceedings. ESO, Germany, p. 233

Herbig G. H., 2008, AJ, 135, 637

Herczeg G. J. et al., 2017, ApJ, 849, 43

Hubber D. et al., 2011a, SEREN: A SPH code for star and planet formation simulations, Astrophysics Source Code Library, record ascl:1102.010.

Hubber D. A., Batty C. P., McLeod A., Whitworth A. P., 2011b, A\&A, 529, A27

Hunter T. R. et al., 2017, ApJ, 837, L29

Johnstone D. et al., 2018, ApJ, 854, 31

Kenyon S. J., Hartmann L. W., Strom K. M., Strom S. E., 1990, AJ, 99, 869

Kóspál Á., Ábrahám P., Prusti T., Acosta-Pulido J., Hony S., Moór A., Siebenmorgen R., 2007, A\&A, 470, 211

Kóspál Á. et al., 2011, A\&A, 527, A133

Kun M., Ábrahám P., Acosta Pulido J. A., Moór A., Prusti T., 2019, MNRAS, 483,4424

MacFarlane B. A., Stamatellos D., 2017, MNRAS, 472, 3775

Mairs S. et al., 2018, Astron. Telegram, 11583, 1

Mathis J. S., Rumpl W., Nordsieck K. H., 1977, ApJ, 217, 425

Mercer A., Stamatellos D., 2017, MNRAS, 465, 2

Myers P. C., Ladd E. F., 1993, ApJ, 413, L47

Ossenkopf V., Henning T., 1994, A\&A, 291, 943

Price D. J., 2007, Publ. Astron. Soc. Aust., 24, 159

Robitaille T. P., 2011, A\&A, 536, A79

Safron E. J. et al., 2015, ApJ, 800, L5

Stamatellos D., Whitworth A. P., 2005, A\&A, 439, 153 
Stamatellos D., Whitworth A. P., Bisbas T., Goodwin S., 2007, A\&A, 475, 37

Stamatellos D., Whitworth A. P., Hubber D. A., 2011, ApJ, 730, 32

Stamatellos D., Whitworth A. P., Hubber D. A., 2012, MNRAS, 427, 1182

Whitney B. A., Wood K., Bjorkman J. E., Wolff M. J., 2003a, ApJ, 591, 1049

Whitney B. A., Wood K., Bjorkman J. E., Cohen M., 2003b, ApJ, 598, 1079
Yoo H. et al., 2017, ApJ, 849, 69

Zhu Z., Hartmann L., Gammie C. F., Book L. G., Simon J. B., Engelhard E., 2010a, ApJ, 713, 1134

Zhu Z., Hartmann L., Gammie C., 2010b, ApJ, 713, 1143

This paper has been typeset from a $\mathrm{T}_{\mathrm{E}} \mathrm{X} / \mathrm{LAT}_{\mathrm{E}} \mathrm{X}$ file prepared by the author. 